\title{
Estudio sobre la divulgación, presencia y prestigio de la Revista de Otorrinolaringología y Cirugía de Cabeza y Cuello, año 2006
}

\author{
Diffusion, presence and prestige of the \\ Revista de Otorrinolaringología y Cirugía de Cabeza y Cuello, 2006
}

Alejandro Peña M.

RESUMEN

\begin{abstract}
Antecedentes: Las revistas médicas requieren, además de una excelente calidad de los trabajos originales o artículos de investigación que en ellas se publiquen, una buena difusión o divulgación de éstos.

Objetivos: El propósito de este estudio es conocer, en este ámbito, la actual realidad que la Revista Otorrinolaringología Cirugía de Cabeza y Cuello alcanza en sus niveles de difusión, presencia y prestigio tanto nacional como internacional.
\end{abstract}

Material y método: Para efectuar esta investigación se procedió al estudio y análisis de algunos de los índices bibliométricos más conocidos: El Factor de Impacto Nacional, las Citas o Referencias Bibliográficas, la indexación en las Bases de Datos Biomédicos, la calidad de difusión de la Revista en formato electrónico vía Internet y el uso del idioma inglés y de los Resúmenes Estructurados o Abstracts.

Resultados: Los resultados del cálculo del Factor de Impacto Nacional para el año 2006, revelaron un índice bajísimo de 0,101. El estudio de las Citas Bibliográficas del año 2006 demostró una abrumadora presencia de revistas norteamericanas (86\%), escasísimas europeas y nacionales, y ninguna de otros países latinoamericanos. El estudio de la indexación a las Bases de Datos Biomédicos, mostró que la revista está registrada sólo en LILACS, la cual es muy poco requerida por los investigadores. La visibilidad de la revista en formato electrónico es baja, puesto que puede ser consultada casi sólo en la página web de la Sociedad Chilena de Otorrinolaringología, carece de versión en inglés, y tampoco publica los resúmenes de los trabajos en Abstract o Resúmenes estructurados.

Conclusiones: El estudio reveló que actualmente la Rev Otorrinolaringol Cir Cabeza Cuello, tiene una mala difusión y presencia tanto nacional como internacional, por lo que parece de urgente necesidad mejorar este aspecto. Primero: a través de la indexación en algunas de las Bases de Datos Biomédicos de mayor prestigio: SciELO. y MEDLINE/ PubMed; segundo: facilitar el acceso de investigadores extranjeros a la revista; tercero: proyectar en un futuro cercano editar la revista de formato electrónico en versión bilingüe (inglés-castellano), como lo hacen ya algunas revistas latinoamericanas de la especialidad, y finalmente, plantear al Comité Editorial la necesidad de agregar al título de la revista la palabra chilena, (Revista Chilena de Otorrinolaringología y Cirugía de Cabeza y Cuello) para una mejor identificación internacional de la publicación

Palabras Clave: Indices Bibliométricos, Bases de Datos Biomédicos, Factor de Impacto.

Otorrinolaringólogo, Talca 


\section{ABSTRACT}

Introduction. Besides an excellent quality of the papers published, medical journals require good diffusion to reach potential readers.

Goal. To investigate the actual situation of the Rev Otorrinolaringol Cir Cabeza Cuello, in terms of levels of national and international diffusion, presence and prestige.

Materials and Methods. Review and analysis of the journal' presence in several of well known bibliometric indexes: National Impact Factor, Citation Reports, indexation in biomedical databases, e-journal rate of diffusion via Internet, existence of an English version and use of Abstracts or summaries.

Results. The calculated National Impact Factor for 2006 was a very low: 0.101. Analyses of Citation Reports for 2006 showed an overwhelming presence of American journals (86\%), a few Chilean and European journals and none from other Latin American countries. Review of biomedical databases indexation showed that the journal is indexed only in LILACS, a database that is not commonly used by researchers. The electronic version of the journal has a low visibility, because it can be only accessed via the website of the Sociedad Chilena de Otorrinolaringología, it does not have an English version and neither Abstracts nor summaries are published.

Conclusions: This study showed that, at the present time, the Rev Otorrinolaringol Cir Cabeza Cuello has a poor diffusion and very little national and international presence. $A$ substantial improvement on this condition seems urgent. Several action lines are proposed: First, indexation in widely used databases, such as SciELO and MEDLINE/PubMed; secondly, make access to the journal by foreign researchers easier; in third place, publish in the near future the electronic version of the journal in both English and Spanish, similar to other Latin American journals on the area; and finally, propose to the Editorial Committee to add the word «Chilena» to the journal title, in order to improve the international identification of the journal.

Key words: Bibliometric indexes, Biomedical databases, Impact factor

\section{INTRODUCCIÓN}

Las cualidades que distinguen a las revistas biomédicas frente a cualquier otro género de literatura científica son, su capacidad de actualización permanente del conocimiento, de ser el medio más inmediato, activo y eficaz de comunicación entre los investigadores y de tener la virtud de propiciar la integración de la información a la propia cultura de la ciencia.

En el mundo existen actualmente alrededor de 60 mil revistas biomédicas en las que aparecen cerca de 75 mil artículos cada semana ${ }^{1}$, sin embargo el $50 \%$ de estos artículos jamás serán citados, lo que hace suponer que se publica más de 10 necesario, 0 que se publican temas poco relevantes, 0 que las revistas tienen escasa 0 mala divulgación o que se editan en idiomas poco conocidos en el mundo científico.

Así los propósitos más importantes de una revista médica serán dar a conocer 0 difundir de la mejor manera posible los trabajos que en ella se publiquen, estimular la creación y excelencia de los artículos de investigación, y de otros tipos, contribuyendo de esta manera al perfeccionamiento continuo de los médicos a través de la lectura y estudio de ellos.

\section{OBJETIVOS}

La finalidad de este estudio ha sido conocer la realidad actual de la Rev Otorrinolaringol Cir Cabeza Cuello, en lo concerniente a su divulgación, presencia y prestigio, a través del análisis e investigación de algunos de los Indices Bibliométricos internacionales más conocidos: el Factor de Impacto Nacional, el estudio de las Citas Bibliográficas, la Indexación en las Bases de Datos Biomédicos, el nivel de difusión de la Revista en formato Electrónico y el uso del idioma Inglés, y de Resúmenes Estructurados en ella. 
Una adecuada divulgación, apreciación y presencia de una revista médica dependerá de varios factores, entre los cuales pueden citarse:

a) Calidad y cantidad de trabajos originales 0 de artículos de investigación.

b) Frecuencia y regularidad en su publicación, mínimo trimestral, como exigen actualmente las Bases de Datos Biomédicos Internacionales $^{2}$, unido a una entrega y distribución oportuna de ella.

c) Incorporación o indexación a los Índices Bibliográficos o Bases de Datos Biomédicos más prestigiados y usados por los investigadores.

d) Publicación de la Revista en formato Electrónico, simultáneamente con la versión impresa, vía Internet, que sea de fácil acceso universal y en texto completo idealmente en versión bilingüe, para una mayor visibilidad de ésta.

e) Presencia en el Comité Editorial de especialistas extranjeros de prestigio.

f) Apertura de la revista a trabajos de investigación de muy buen nivel de autores extranjeros.

\section{Factor de impacto nacional}

Ha sido una constante de las revistas médicas especialmente las anglosajonas conocer el prestigio de ellas, basándose principalmente en la frecuencia con la cual los clínicos o investigadores citan los trabajos publicadas en dichas revistas; así a mayor frecuencia de citación aumentaría su prestigio, lo cual a su vez llevaría a que los médicos se interesaran en publicar sus mejores trabajos en aquellas revistas más citadas y supuestamente más leídas ${ }^{3}$.

Este hecho a su vez permitiría a los Editores, seleccionar los trabajos de más interés o calidad, aumentando la frecuencia de rechazos con lo que se privilegia la excelencia de artículos.

Alrededor del año 1962, Garfield y Shear fundaron en Filadelfia el ISI (Institute of Scientific Investigation $)^{4}$, y empezaron a publicar el SCl (Scientific Citation Index) o (Thomson Scientific) en Inglaterra, el cual reúne y codifica las publicaciones periódicas científicas más importantes, alcanzando actualmente más de 5.800 revistas, la inmensa mayoría en inglés, correspondiendo de ellas, al área biomédica 1.500 , también la mayoría en inglés, índice en el cual están incorporadas sólo dos revistas médicas chilenas.
Basados en la información recogida del $\mathrm{SCl}$, Garfield y col comenzaron a publicar en el año 1972 el llamado Journal Impact Factor o JIF que a pesar de ser muy criticado ha sido aceptado ampliamente en el área biomédica como supuesto índice de calidad de las revistas, aunque dicho índice no significa necesariamente que aquellas con un buen puntaje, sean las más leídas o consultadas.

Para que una revista sea incluida en el Journal IF, es necesario que la publicación esté indexada en el exigente $\mathrm{SCl}$. En la esfera otorrinolaringológica, están incorporadas sólo 29 revistas; 27 en idioma inglés, una en alemán y otra en francés. Del total: 21 revistas son norteamericanas y sólo 6 europeas ${ }^{5}$.

Este Impact Factor se calcula anualmente de la siguiente manera: Para un período de tres años, se contabilizan primero las citas bibliográficas del tercer año de la revista por ej. 2006, que se refieran a artículos publicados en la misma revista los dos años precedentes por ej. 2004 y 2005, la cantidad de citas se coloca como numerador y se divide por el número de artículos publicados en estos dos años por ej. 2004 y 2005, número que se coloca como denominador, su cuociente es el llamado Impact Factor ${ }^{4}$.

Considerando el hecho que el número de revistas médicas en idioma español indexadas en el SCl es escasísimo, el Instituto de Historia de la Ciencia y Documentación López Piñero de España, creó el llamado Factor de Impacto Nacional en el cual están registradas alrededor de 100 revistas médicas españolas ${ }^{6}$, igual idea surgió en la Asociación Chilena de Editores de Revistas Biomédicas en su quinto Simposium, moción que aún no ha sido concretada?

Al efectuar este cálculo del Factor de Impacto Nacional en la Rev Otorrinolaringol Cir Cabeza Cuello para el año 2006, los resultados fueron los siguientes: en los tres números del volumen 66 (año 2006), se publicaron 420 citas 0 referencias bibliográficas, de estas citas sólo 6 , se referían a artículos publicados en la revista los dos años precedentes, 2004 y 2005; en estos dos años se publicaron 59 artículos, la división de estas dos cifras determina un cuociente de 0,101 que sería el Factor de Impacto Nacional 2006 para la revista de otorrinolaringología de nuestro país. A modo de 
comparación, el Impact Factor del año 2003 de las revistas norteamericanas otorrinolaringológicas más leídas o consultadas fue el siguiente: Annals of Otology, Rhynology and Laryngology 1,067; Archives of Otolaryngology-Head and Neck Surgery 1,408; Laryngoscope 1,576; y Otolaryngology- Head and Neck Surgery 1,3005. La Revista Médica de Chile, obtuvo para el año 2005 un Impact Factor de 0,3538.

El bajo Factor de Impacto Nacional de la Rev Otorrinolaringol Cir Cabeza Cuello puede atribuirse a varios motivos: 1 . Los artículos originales pudieran ser poco relevantes y/0 importantes; 2. La revista posiblemente sea poco leída 0 consultada por los autores nacionales; y 3 . Por tratarse de una revista cuatrimestral, los trabajos originales de referencia para los potenciales investigadores posiblemente sean escasos.

El SCI administrado y dirigido por el ISI, institución privada supuestamente sin fines de lucro ${ }^{9}$, ha sido objeto de muchas críticas, pues el Impact Factor puede ser manipulado y manejado maliciosamente y detrás de ello estarían las grandes empresas editoriales lo que explicaría el alto costo que tiene la suscripción a algunas publicaciones cuyo Impact Factor es elevado, además del subido precio que alcanzan estas revistas al ser solicitados, algunos de sus artículos, vía Internet ${ }^{10}$.

El Impact Factor puede ser modificado o manejado, por ejemplo, a través de autocitas o por la publicación de extensos artículos de revisión en que cita a la revista en cuestión etc.

A su vez, los editores de revistas médicas preferirían recibir y publicar aquellos trabajos en cuya bibliografías se cita frecuentemente la revista ${ }^{11-13}$.

Tampoco el Impact Factor, necesariamente, representa a las revistas de mayor prestigio 0 calidad científica, pues, habitualmente, las revistas de ciencias básicas 0 de investigación tienen un alto Impact Factor, debido a que su campo de estudio es habitualmente más estrecho de manera que tienen un alto índice de autocitas. Así, por ejemplo, en la esfera otorrinolaringológica, las tres revistas que tienen el mayor Impact Factor, no son las más consultadas por los médicos clínicos, sino por los investigadores de fisiología o fisiopatología de la especialidad: JARO-Journal of the Association for Research in Otolaringology 2,316; Head and Neck Journal for the Science and Specialities of the Head and Neck 2,220; y Ear and Hearing $2,302^{5}$.

Por otra parte, los artículos de autores latinoamericanos de las revistas indexadas en el SClThomson Scientific, representaban en el año 1999 sólo el 1,8\% del total. El $85 \%$ de estos artículos provenían de sólo cuatro países: Brasil, México, Argentina y Chile. Estos artículos fueron citados $50 \%$ menos que el promedio mundial para trabajos del mismo campo, posiblemente por las barreras idiomáticas de los idiomas español y portugués ${ }^{14}$.

\section{CITAS O REFERENCIAS BIBLIOGRÁFICAS}

Las referencias bibliográficas son elegidas por los autores de los artículos para darle sustento a sus trabajos: a su origen, a su propósito y eventual hipótesis, a su diseño metodológico, a los resultados e interpretación que éstos le inspiraron. Las referencias bibliográficas reflejan una de las características de la investigación científica: cada nuevo trabajo se apoya en los de los predecesores en ese campo. En otros término, la bibliografía muestra los fundamentos externos a que recurren lo autores para cada una de las aseveraciones de sus artículos, para relacionarlos con otros trabajos y comparar sus resultados con los comunicados previamente por otros. Su selección adecuada es indispensable para la fortaleza del estudio y la credibilidad del escrito.

En síntesis, la presentación de las referencias exige un esfuerzo de selección, dando preferencia a la originalidad y trascendencia de las publicaciones citadas y a su estricta relación con el aspecto del tema que se está abordando $0^{15}$.

En al año 2006 (volumen 66), la Revista de Otorrinolaringología y Cirugía de Cabeza y Cuello publicó en sus 34 artículos un total de 420 citas bibliográficas. El análisis de estas citas reveló que 385 correspondieron a revistas, y 35 a libros 0 capítulos de libros o comunicaciones 0 seminarios etc. De las 385 citas de revistas, 91\% correspondieron a publicaciones en idioma inglés (350 citas); sólo $7,5 \%$ a revistas publicadas en idioma español ( 29 citas); y $1,5 \%$ en otros idiomas: alemán y francés (6 citas).

De las revistas citadas en español, 13 correspondieron a la Rev Otorrinolaringol Cir Cabeza 
Cuello (3\%), 8 a otras revistas chilenas y 8 al Acta Otorrinolaringológica española.

De las revistas citadas en idioma inglés la inmensa mayoría correspondió a revistas norteamericanas (86\%), encabezadas por Archives of Otolaryngology, Laryngoscope y Otolaryngology Head and Neck Surgery .

Del análisis de estas cifras llamó la atención la aplastante presencia norteamericana en la otorrinolaringología chilena, que no es sólo privativo de Chile sino que también del resto de Latinoamérica, e incluso de Cuba en que el $75 \%$ de las citas de la revista médica más leída en Cuba (Revista Cubana de Medicina General Integral) corresponden a revistas estadounidenses ${ }^{1}$.

Este paternalismo de la medicina norteamericana en Latinoamérica no corresponde, sin embargo, a la realidad internacional. Así, la información dada para el año 2002 por el ISI reveló que el 33\% de todos los artículos médicos provenían de EE.UU., seguido por Japón (9,4\%), Reino Unido (8,9\%),y Alemania (8,6\%). En Latinoamérica, Brasil encabezaba esta estadística con el 1,5\% ${ }^{16}$.

En el campo otorrinolangológico, la realidad es similar. Un estudio de Cimino ${ }^{17}$, demostró que en el año 2006, 38\% de los trabajos provenían de EE.UU. y $36,1 \%$ de Europa. Del total europeo, $28,5 \%$ correspondía al Reino Unido; 26,2\% a Alemania; $7,2 \%$ a Italia; $5,8 \%$ a Suecia; $5,5 \%$ a Francia; $4,9 \%$ a Holanda y $4 \%$ a España; a la luz de estos porcentajes, debe sin embargo señalarse, que algunos investigadores europeos y también japoneses prefieren publicar sus experiencias en revistas norteamericanas, buscando una mayor difusión.

La exposición de esta realidad llevaría a pensar que los otorrinolaringólogos chilenos sólo tendrían acceso a la literatura norteamericana, ignorando las experiencias europeas y las del resto del mundo, quizás por dificultadas para obtener revistas europeas 0 asiáticas o por barreras idiomáticas.

En las 385 citas bibliográficas de revistas, no existe ninguna latinoamericana, ignorando, por ejemplo, a Brasil, en circunstancias que la Revista Brasileira de Otorrinolaringología, es más antigua que la chilena, editándose bimestralmente desde 1934, con amplia difusión en formato electrónico, con versión en inglés e indexación en cuatro Bases de Datos Biomédicos de prestigio ${ }^{18}$.

\section{BASES DE DATOS BIOMÉDICOS O ÍNDICES BIBLIOGRÁFICOS INTERNACIONALES}

Uno de los requisitos más importantes para una adecuada difusión y presencia de una revista médica es su ingreso o incorporación a las Bases de Datos Biomédicos de mayor prestigio y alcance internacional. La importancia de incluir una revista en índices bibliográficos internacionales tiene ventajas capitales: le otorga una mayor visibilidad y por ende una mayor diseminación nacional e internacional y permite un control bibliográfico de la producción científica.

Un estudio nacional (2003), demostró que de 37 revistas médicas encuestadas $19 \%$ no estaba indexada en ninguna Base de Datos Biomédicos; $81 \%$ lo estaba en LILACS; $13,5 \%$ lo estaba en MEDLINE/PubMed, y sólo dos estaban indexadas en el SCl${ }^{2}$.

La Rev Otorrinolaringol Cir Cabeza Cuello está, actualmente, sólo indexada en LILACS (Literatura Latinoamericana y del Caribe en Ciencias de la Salud), ésta base de datos es muy poco consultada incluso por los propios latinoamericanos ${ }^{19}$, esto contrasta con la Revista Médica de Chile una de las más antiguas (123 años) y prestigiadas del continente, que está indexada en 10 Bases de Datos Biomédicos ${ }^{9}$; por consiguiente constituye una labor importante del Comité Editorial de la revista registrar la publicación en Bases de Datos de mayor alcance y trascendencia internacional.

Un primer paso, debería ser incorporar la revista a SciELO (Scientific Electronic Library on Line), biblioteca electrónica que incluye una colección seleccionada de revistas latinoamericanas y de España; este proyecto está siendo dirigido en Chile por CONICYT, y contempla el desarrollo de una metodología común para la preparación, almacenamiento, diseminación y evaluación de la literatura científica en formato electrónico, facilitando el acceso libre y en texto completo de todas las revistas indexadas en SciELO ${ }^{20}$, a su vez, y como complemento muy importante, las revistas de la Salud incorporadas en SciELO, tienen enlace directo con LILACS y MEDLINE/PubMed, ésta última es propiedad del gobierno de los EE.UU., y es actualmente la Base de Datos Biomédicos más grande del mundo; producida por la US. Nacional Library of Medicine $y$ el National Institutes of Health, 
contiene más de 16 millones de registros de la literatura biomédica desde 1950, con referencias bibliográficas de más de 4.800 revistas biomédicas de EE.UU. y de otros 70 países, y con actualizaciones mensuales permanentes de la información ${ }^{21}$, permitiendo realizar búsquedas mediante palabras claves 0 encabezados de temas médicos, conocidos como MeSH (Medical Subject Heading); estos términos forman un glosario controlado que se utiliza para garantizar que la indexación de los artículos acerca de un tema específico sea coherente.

La revista de la Oficina Panamericana de la Salud, publicó en el año 2005, un artículo sobre el uso de las Bases de Datos Biomédicos de investigadores hispanoamericanos, principalmente de México, Argentina y Chile y cuales eran las más usadas en sus investigaciones, los resultados fueron los siguiente: la más requerida fue MEDLINE/ PubMed con $35 \%$, seguido por buscadores generales tales como Google, y Yahoo con $16 \%$, en cambio BIREME /LILACS era usado por sólo $6 \%$ de Ios encuestados ${ }^{19}$.

\section{REVISTA EN FORMATO ELECTRÓNICO}

Uno de los mecanismos actuales más importantes para una adecuada difusión de las revistas médicas es la edición de éstas en formato electrónica vía Internet, y mejor aún si esta publicación se hace en texto completo, sin costo y preferentemente en idioma inglés, así lo han comprendido la mayor parte de las revistas de otorrinolaringología norteamericanas y europeas, que publican sus ediciones vía Internet, algunas sin costo, pero la mayoría con un prepago, que es necesario para acceder a la lectura de ellas.

Actualmente la Rev Otorrinolaringol Cir Cabeza Cuello es accesible en formato electrónico principalmente a través de la pagina web de la Sociedad Chilena de Otorrinolaringología, siendo así difícil de encontrar para investigadores extranjeros, sin embargo al indexar la revista a SciELO y estar ésta en enlace con MEDLINE/PubMed, les será muy fácil a clínicos y médicos extranjeros poder conocer y leer la revista en texto completo, surgiendo inmediatamente otra necesidad, consistente en que todos los trabajos que se publiquen en ella deben tener un resumen estructurado 0 abstract, el cual es un requisito indispensable para la indexación de los trabajos en estos índices bibliográficos internacionales, y que es una medida que la revista no está actualmente exigiendo a los médicos que publican en ella.

\section{TÍTULO DE LA REVISTA}

Los fundadores de la Revista de Otorrinolaringología y Cirugía de Cabeza y Cuello, quizás jamás pensaron que ésta pudiera llegar a tener una difusión que traspasara nuestras fronteras, así el problema actual estriba en que cualquier médico extranjero que se interese en algún trabajo publicado en la revista, le será difícil conocer el país de origen de ésta, en tal sentido en algún momento el Comité Editorial deberá decidir agregar al nombre de la revista, la palabra "chilena», pudiendo así llamarse en un futuro cercano Revista Chilena de Otorrinolaringología y Cirugía de Cabeza y Cuello, para poder tener una mayor presencia y visibilidad internacional.

\section{REVISTA BILINGÜE}

Hoy en día, el idioma inglés es la lengua franca de las Ciencias, llegando a ser la forma internacional para difundir las Ciencias Biomédicas, así lo revelan por ejemplo las propias cifras de las citas bibliográficas de la Rev Otorrinolaringol Cir Cabeza Cuello del año 2006, en que el $91 \%$ de estas citas fueron referencias en idioma inglés, y ésta es una realidad no sólo nacional sino mundial ${ }^{22,23}$. Al respecto y a mayor abundamiento, el estudio de Beníte $^{14}$, señalaba que un trabajo publicado en español, será $50 \%$ menos citado que un trabajo de igual calidad publicado en inglés.

Así lo han comprendido la mayor parte de los editores de las revistas otorrinolaringológicas de prestigio de países no angloparlantes, tanto de Europa como de Latinoamérica quienes publican sus revistas particularmente en su formato electrónico en idioma inglés, lo hacen actualmente en Latinoamérica los Anales Mexicanos de Otorrinolaringología y la Revista Brasileira de Otorrinolaringologia, y en España, el Acta Española de 
Otorrinolaringología; estos hechos deberán llevar en algún momento al Comité Editorial de la revista, a plantearse la necesidad de publicar una edición bilingüe en su formato electrónico, buscando mejorar su visibilidad y prestigio.

\section{CONCLUSIONES}

Del estudio y análisis de los índices bibliométricos, se desprende que la revista tiene un bajo perfil particularmente en lo referente a su difusión tanto internacional como también nacional, de manera que parece importante además de luchar por mejorar la calidad de los trabajos que en ella se publiquen darle una mayor presencia 0 visualización internacional a la publicación.

Para ello, es necesario primero: junto con aumentar las exigencias a los trabajos que se envían para su publicación buscando la excelencia, una mejor difusión de éstos artículos a través de su pronta indexación en SciELO y eventualmente MEDLINE/PubMed; segundo: mejorar la divulgación de la revista de formato electrónico incorporando la publicación a SciELO, y exigiendo a los autores la publicación de sus trabajos con resúmenes estructurados (abstract); tercero: plantear en un futuro cercano la necesidad de publicar la revista de formato electrónico en forma bilingüe para lograr una mayor notoriedad internacional; finalmente, y por la misma razón, debiera discutirse la necesidad de cambiar el nombre de la revista, reemplazándolo por el de Revista Chilena de Otorrinolaringología y Cirugía de Cabeza y Cuello lo que contribuiría también a mejorar su presencia y prestigio internacional.

\section{BIBLIOGRAFÍA}

1. López A, Díaz S. Alcance de las referencias bibliográficas en la Revista Cubana de Medicina General Integral. Rev Cubana Med Gen Integr 1996; 12(1): 35-8.

2. Oyarzún M. Ingreso de las revistas biomédicas nacionales a los índices bibliográficos internacionales: Desafíos y oportunidades. Rev Chil Enf Respir 2003; 19:5-7.
3. Rodríguez M. Finalidad última de una Revista Médica (editorial). An ORL Mex 2006; 51(1): 1-2.

4. Garfield E. The history and meaning of the Journal Impact Factor. JAMA 2006; 295: 90-3.

5. Impact Factor: Science Citation IndexOtorhinolaryngology 2003. Disponible en: www.ent.com.cn/magazine/sci journal/imf 2003.html.

6. Factor de Impacto Potencial de las Revistas Médicas Españolas. Inst Historia de la Ciencia y Documentación López Piñero. Disponible en: http//ime.uv.es/imecitas/factor impacto.shtml.

7. Oyarzún M. Como atraer más y mejores manuscritos para publicación en las revistas biomédicas chilenas. Rev Méd Chile 2002; 130(3): 267-74.

8. ReYes H. El Aniversario 135 de la Revista Médica de Chile. Rev Méd Chile 2007; 135 (1): 7-10.

9. Impact Factor, from Wikipedia. Disponible en: http://en.wikipedia org/wiki/impact Factor.

10. Figa-Talamanca A. L'Impact Factor nella valutazione della ricerca e nello sviluppo dell'editoria scientifica. Disponble en: http:// siba2.unile.it/sinm/4sinm/interventi/figtalam.htm.

11. HA TC, TAN SB, Soo KC. The Journal Impact Factor: too much of an impact? Ann Acad Med Singapore 2006; 35(12): 911-6 .

12. Somnath S. Impact Factor: a valid measure of journal quality? J Med Lib Assoc 2003; 91 (1): 42-6.

13. The Plos Medicine Editors. The Impact Factor game. Plos Med 2006; 3(6):e 291.

14. Benitez Bribiesca H. The ups and downs of the Impact Factor. Arch Med Res Mex 2000; 33(2): 91-4.

15. ReYes H. Las referencias en artículos publicados en revistas biomédicas. Rev Méd Chile 2001; 129(4): 343-5.

16- Rodríguez M, Montiel F. The medical investigation in Mexico. An ORL Mex 2006; 51(2): 55-6.

17. Cimino $M$, Tiziana M, Donatella $U$. Trends in otolaryngology research during the period 19952000: a bibliometric approach. OtolaryngologyHead and Neck Surgery 2005: 132 (2): 295-302.

18. Revista Brasileira de Otorrinoalringologia. Disponible en: http://www.rborl.orgbr/portugues/ default.asp 
19. Ospina E, Reveitz-Herault L, Cardona A. Uso de las bases de datos bibliográficos por investigadores biomédicos latinoamericanos hispanoparlantes: estudio transversal. Rev Panam Salud Pública 2005; 17(4):230-6.

20. SciELO (Scientific Electronic Library on Line) Disponible en: http://scielo.cl/scielo php

21. MEDLINE/PubMed Visita guiada. Disponible en: http://escuela med puc.cl/recursos/MBE 2001/guiadamedline.htm/
22. Arribalzaga E. El idioma y el factor de impacto de las revistas científicas. South Am J Thorac Surg 2000; 6(2): 25-9.

23- Reyes H, Kauffman R, Andrensen M. Tradición y Progreso: ¿Ahora también una revista bilingüe? Rev Méd Chile 2000; 128 (11): 1187-9. 